\title{
Microstrip line ferromagnetic resonance and Brillouin light scattering investigations of magnetic properties of $\mathrm{Co}_{2} \mathrm{MnGe}$ Heusler thin films
}

\author{
M. Belmeguenai, ${ }^{1,2}$ F. Zighem, ${ }^{1}$ Y. Roussigné, ${ }^{1}$ S-M. Chérif, ${ }^{1}$ P. Moch, ${ }^{1}$ K. Westerholt, ${ }^{3}$ G. Woltersdorf, ${ }^{2}$ and G. Bayreuther ${ }^{2}$ \\ ${ }^{1}$ LPMTM, Institut Galilée, UPR 9001 CNRS, Université Paris 13, 99 Avenue Jean-Baptiste Clément, F-93430 Villetaneuse, France \\ ${ }^{2}$ Institut für Experimentelle Physik, Universität Regensburg, Universitätsstraße 31, 93040 Regensburg, Germany \\ ${ }^{3}$ Institut für Experimentelle Physik/Festkörperphysik, Ruhr-Universität Bochum, 44780 Bochum, Germany
}

(Received 10 July 2008; revised manuscript received 2 November 2008; published 16 January 2009)

\begin{abstract}
$\mathrm{Co}_{2} \mathrm{MnGe}$ films of different thicknesses $\left(34,55\right.$, and $83 \mathrm{~nm}$ ) were grown by rf sputtering at $400{ }^{\circ} \mathrm{C}$ on single-crystal $\mathrm{Al}_{2} \mathrm{O}_{3}$ corundum substrates showing an in-plane $\mathbf{c}$ axis. Their dynamic magnetic properties were studied using conventional and microstrip line (MS) ferromagnetic resonances (FMRs), as well as Brillouin light scattering (BLS) techniques. The effective magnetizations and gyromagnetic factors are first deduced from the resonance spectra involving the uniform magnetic mode under in-plane and out-of-plane magnetic applied fields. The angular dependence of the frequency, measured under a weak in-plane magnetic applied field, then allows deriving of the parameters describing the in-plane magnetic anisotropy. In the 34- and 55-nm-thick films, its behavior is described assuming a magnetic energy density showing an orthorhombic symmetry with a twofold axis normal to the film and planar anisotropy axes at $\pm \pi / 4$ of the $\mathbf{c}$ axis of the substrate; however, due to the comparative deduced values of the pertinent coefficients, this energy density is predominantly tetragonal. In the thickest film $(83 \mathrm{~nm})$, one of the planar anisotropy axes is parallel to $\mathbf{c}$ and approximate tetragonal symmetry is no more observed. Moreover, the orthorhombic symmetry is not completely fulfilled and a small misalignment between the principal directions connected to the uniaxial and the fourfold energy terms appears. Finally, the perpendicular surface standing modes, which are observed in MS-FMR and in BLS spectra, allow evaluation of the exchange stiffness constant. Good agreement between BLS and MS-FMR measurements has been found.
\end{abstract}

DOI: 10.1103/PhysRevB.79.024419

PACS number(s): 76.50.+g, 78.35.+c, 75.30.Gw, 75.40.Gb

\section{INTRODUCTION}

Half-metallic ferromagnets (HMFs) are materials where one of the spin channels is metallic and the second one is insulating. These materials show full spin polarization at the Fermi level and have triggered a lot of interest due to their potential use in spintronic applications. Several HMFs have been predicted by means of electronic structure calculations, for instance, some Heusler alloys such as $\mathrm{Co}_{2} \mathrm{MnGe}$. These materials are considered to be promising candidates for injecting a spin-polarized current from a ferromagnet into a semiconductor and for elaborating ferromagnetic electrodes used in spintronic devices such as magnetic random access memories (MRAMs) since their Curie temperature is high (905 K) (Ref. 1) and since they show a good lattice matching with the GaAs semiconductor family. ${ }^{2}$ Therefore, great attention has been given in recent years to Heusler alloys. It has been shown that high-quality epitaxial layers of $\mathrm{Co}_{2} \mathrm{MnGe}$ can be grown on $\mathrm{GaAs},{ }^{3}$ which is promising for integration of some spintronic devices with those of the conventional electronics on the same chip. The structural properties and some magnetic characteristics of $\mathrm{Co}_{2} \mathrm{MnGe}$ thin films deposited on $\mathrm{MgO}$ substrates were addressed by Ishakawa et al. ${ }^{4}$ Domain patterns and magnetization rotation in single-crystal (001) $\mathrm{Co}_{2} \mathrm{MnGe}$ films epitaxially grown on GaAs (001) substrates have been studied using the magneto-optical indicator film imaging technique. ${ }^{5}$ In addition, interlayer exchange coupling in trilayers, which consist of full-Heusler $\mathrm{Co}_{2} \mathrm{MnSi}$ as ferromagnetic layers separated by a thin $\mathrm{Cr}$ spacer layer, has been proved. ${ }^{6}$ Furthermore, a large tunnel magnetoresistance (TMR) effect has been measured recently by several groups in magnetic tunnel junctions with full-Heusler alloy electrodes, ${ }^{7-9}$ proving experimentally their half metallicity.

Despite this intense research activity on Heuslers, which is mainly focused on their structural properties and on the way to improve the tunnel magnetoresistance, the static and dynamic magnetic properties of such alloys remain less explored. ${ }^{10}$ The dynamics of these materials within the $1-10$ $\mathrm{GHz}$ frequency range, which determines the high-speed response, is a key for their future technologic applications, especially in view of increasing data rates in magnetic storage devices. Moreover, small magnetic damping constants are strongly requested for MRAM, in which the control of the magnetization is carried out using current where switching current density is proportional to the damping value. ${ }^{11}$ Therefore, the aim of this paper is to investigate anisotropies and dynamic magnetic properties of $\mathrm{Co}_{2} \mathrm{MnGe}$ thin films.

In this paper, in addition to static magnetometric measurements, we used conventional ferromagnetic resonance (FMR), microstrip line FMR (MS-FMR) spectroscopy, and Brillouin light scattering (BLS) for complementary studies of the various excited spin waves, allowing a full magnetic characterization of $\mathrm{Co}_{2} \mathrm{MnGe}$ thin films. The paper is organized as follows: we first define the model to be used for the analysis of our measurements (Sec. II). Section III introduces the samples and the experimental setups used for this study. Section IV starts by summarizing the main static magnetic characteristics of the samples using magneto-optical Kerr effect (MOKE) and then it presents dynamic measurements obtained using FMR, MS-FMR, and BLS. Magnetic parameters which satisfactory fit the FMR and BLS results are then derived. In Sec. V, conclusions are drawn. 


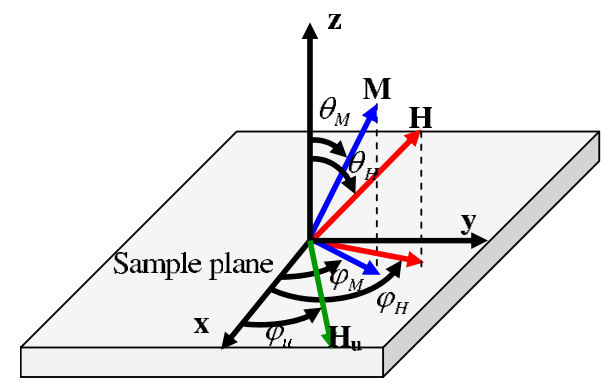

FIG. 1. (Color online) Sketch of the coordinate system used. $\varphi_{M}$ $\left(\varphi_{H}\right)$ is the in-plane angle between the magnetization $\boldsymbol{M}$ (the external field $\boldsymbol{H}$ ) and the $x$ axis, while $\theta_{M}\left(\theta_{H}\right)$ is the out-of-plane angle between the magnetization (the external field $H$ ) and the $z$ axis and $\varphi_{u}$ is the angle of in-plane anisotropy axes $\left(H_{u}\right.$ for the uniaxial anisotropy and $H_{4}$ for the fourfold) with the $x$ axis. $H_{u}=2 K_{u} / M_{s}$ is the uniaxial in-plane anisotropy field.

\section{MODEL AND DISPERSION RELATIONS FOR THE MAGNETIC MODES}

In order to interpret our experimental results on the angular dependence of the resonance frequencies of the various excited spin waves, these frequencies [for the uniform precession mode and for perpendicular standing spin waves (PSSWs)] will be derived in this section as a function of the applied static field magnitude and direction. The system to be used is sketched in Fig. 1. $\varphi_{M}\left(\varphi_{H}\right)$ is the in-plane angle between the magnetization $\boldsymbol{M}$ (the external applied field $\boldsymbol{H}$ ) and the $x$ axis, while $\theta_{M}\left(\theta_{H}\right)$ is the out-of-plane angle between the magnetization (the external field $\boldsymbol{H}$ ) and the normal to the sample plane ( $z$ axis); $\varphi_{u}$ is the angle of one of the in-plane anisotropy axes (see below) with the $x$ axis.

The free-energy density $E$ of a single film is given by

$$
E=E_{\text {Zeeman }}+E_{\text {exch }}+E_{\text {dem }}+E_{\text {anis }} .
$$

The three first terms stand for the Zeeman, the exchange, and the dipolar demagnetizing contributions, respectively, and are expressed in the usual way versus the magnetization $\boldsymbol{M}$, its space derivatives, the applied field $\boldsymbol{H}$, and the stiffness exchange constant $A_{\text {ex }}$. The last term represents the anisotropy energy density and, in the following, its expression derives from the development to fourth order of the cosine directors of the magnetization. In principle, this expression is dictated by symmetry considerations. The bulk magnetic $\mathrm{Co}_{2} \mathrm{MnGe}$ alloy shows a face-centered $L 2_{1}$ cubic structure (space group $F m \overline{3} m$ ). The $\mathbf{c}$ axis of the corundum substrate lies in the layer plane ( $x$ axis). It results, as experimentally verified through x-ray diffraction (XRD) [see Fig. 2(a)], that the [110] axis of the magnetic film is normal to this plane ([110]\| $z$ axis). This suggests describing the anisotropy contribution using an expression reflecting an orthorhombic symmetry with one twofold axis along $z$. It is convenient to write, using spherical coordinates (see Fig. 1)
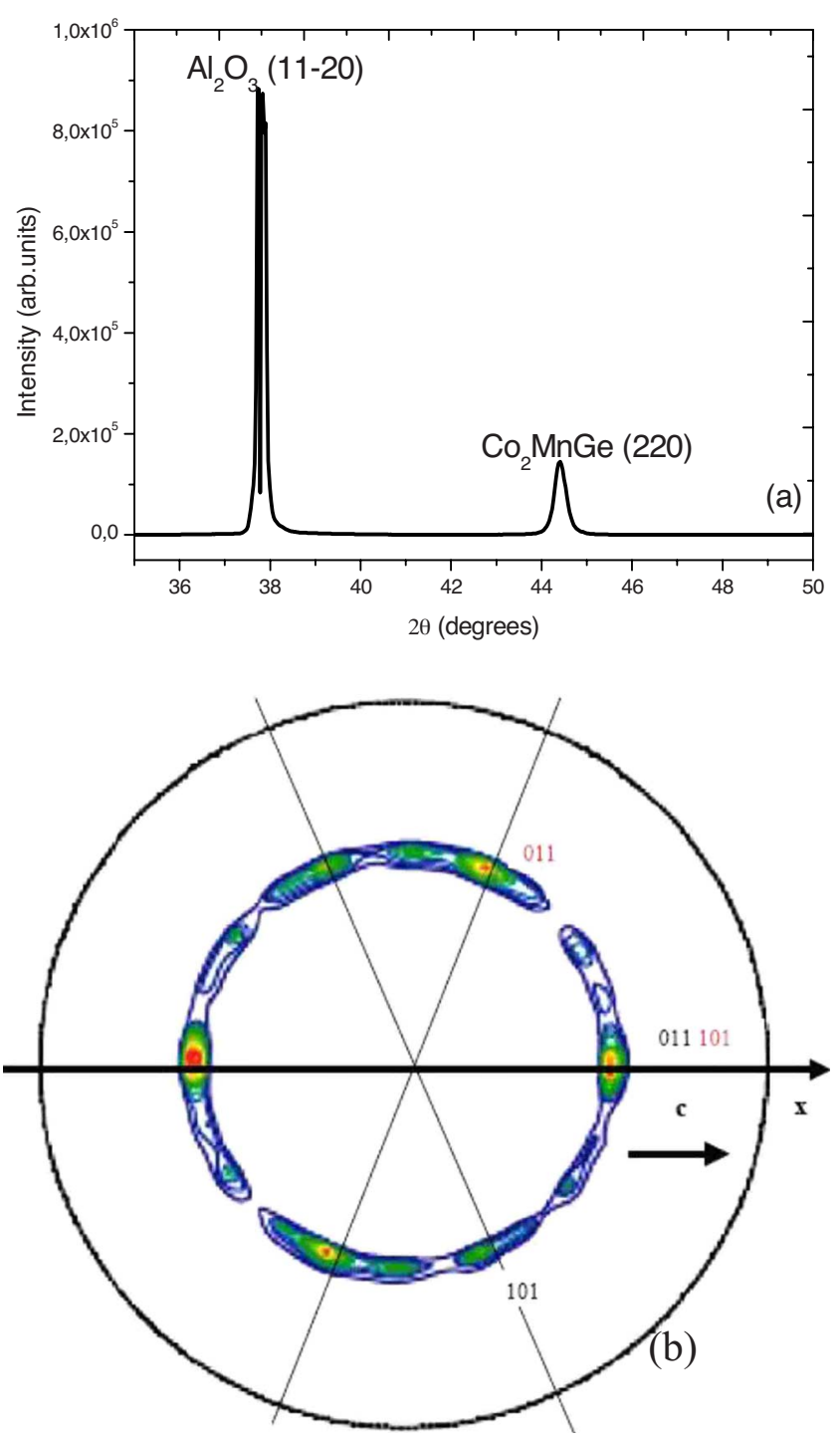

FIG. 2. (Color online) (a) X-ray Bragg scan using $\mathrm{Cu} K \alpha_{1}$ radiation and (b) partial $\mathrm{X}$-ray pole figure for the 55-nm-thick $\mathrm{Co}_{2} \mathrm{MnGe}$ sample.

$$
\begin{aligned}
E_{\text {anis }}= & \left(K_{\perp}-K_{4 \perp}\right) \sin ^{2} \theta_{M}+\frac{K_{4 \perp}}{2} \sin ^{4} \theta_{M} \\
& -\frac{K_{4}}{8} \sin ^{4} \theta_{M}\left[3+\cos 4\left(\varphi_{M}-\varphi_{u}\right)\right] \\
& -\frac{1}{2}\left(K_{u} \sin ^{2} \theta_{M}+K_{u^{\prime}} \sin ^{4} \theta_{M}\right)\left[1+\cos 2\left(\varphi_{M}-\varphi_{u}\right)\right] .
\end{aligned}
$$

The coefficients $K_{u}$ and $K_{u^{\prime}}$ ensure the orthorhombic behavior: their cancellation leads to a tetragonal symmetry. On the other hand, the two first terms provide out-of-plane uniaxial contributions: the second-order out-of-plane uniaxial anisotropy constant $K_{\perp}$ is generally significantly larger than the fourth order one $K_{4 \perp} \cdot{ }^{12}$ It results that the experimental evaluation of $K_{4 \perp}$ from our experimental data is not possible: for simplicity we then state $K_{4 \perp}=0$. The perpendicular aniso- 
tropy field $H_{a \perp}$ is then defined as $H_{a \perp}=2 K_{\perp} / M_{s}$ (where $M_{s}$ is the magnetization at saturation). Moreover, it appears that the available geometrical setup does not allow to discriminate between the contributions relative to $K_{u}$ and to $K_{u^{\prime}}$ since the measured frequencies practically only depend on $\left(K_{u}\right.$ $\left.+K_{u^{\prime}}\right)$ : in view of comparison with previously published analogous determinations, our analysis is done assuming $K_{u^{\prime}}=0$. Finally, we write

$$
\begin{aligned}
E_{\text {anis }}= & K_{\perp} \sin ^{2} \theta_{M}-\frac{1}{2}\left[1+\cos 2\left(\varphi_{M}-\varphi_{u}\right)\right] K_{u} \sin ^{2} \theta_{M} \\
& -\frac{1}{8}\left[3+\cos 4\left(\varphi_{M}-\varphi_{u}\right)\right] K_{4} \sin ^{4} \theta_{M},
\end{aligned}
$$

where $K_{u}$ and $K_{4}$ are the in-plane uniaxial and the fourfold anisotropy constants, respectively. It will be experimentally shown that in the case of thin enough films (55 nm or less) the angle $\varphi_{u}$ between one of the in-plane uniaxial anisotropy axes and the $x$ axis is equal to $\pi / 4$.

The total-energy density contribution to consider in the following is now given by

$$
\begin{aligned}
E= & -M_{s} H\left[\sin \theta_{M} \sin \theta_{H} \cos \left(\varphi_{M}-\varphi_{H}\right)+\cos \theta_{M} \cos \theta_{H}\right] \\
& -\left(2 \pi M_{s}^{2}-K_{\perp}\right) \sin ^{2} \theta_{M} \\
& -\frac{1}{2}\left[1+\cos 2\left(\varphi_{M}-\varphi_{u}\right)\right] K_{u} \sin ^{2} \theta_{M} \\
& -\frac{1}{8}\left[3+\cos 4\left(\varphi_{M}-\varphi_{u}\right)\right] K_{4} \sin ^{4} \theta_{M},
\end{aligned}
$$

where the exchange energy is omitted. The in-plane and outof-plane equilibrium positions of the magnetization under the applied field are determined by the energy minima.

The resonance frequency $F_{r}$ of the uniform precession mode evaluated at the equilibrium can be obtained from the energy density as follows: ${ }^{13,14}$

$$
F_{r}^{2}=\left(\frac{\gamma}{2 \pi}\right)^{2} \frac{1}{M_{s}^{2} \sin ^{2} \theta_{M}}\left[\frac{\partial^{2} E}{\partial \theta_{M}^{2}} \frac{\partial^{2} E}{\partial \varphi_{M}^{2}}-\left(\frac{\partial^{2} E}{\partial \theta_{M} \partial \varphi_{M}}\right)^{2}\right],
$$

where the derivatives are evaluated for the equilibrium direction of the magnetization and where $\gamma=g \times 8.78$ $\times 10^{6} \mathrm{~Hz} / \mathrm{Oe}$ is the appropriate gyromagnetic factor related to the effective $g$-Landé coefficient.

In the case of an in-plane applied magnetic field, both the magnetization and the applied magnetic field lie in the plane of the film: $\theta_{M}=\theta_{H}=90^{\circ}$. The in-plane resonance frequency $F_{\|}$is given by

$$
F_{\|}=\frac{\gamma}{2 \pi}\left(H_{a} H_{b}\right)^{1 / 2},
$$

where

$$
\begin{aligned}
H_{a}= & H \cos \left(\varphi_{H}-\varphi_{M}\right)+\frac{2 K_{4}}{M_{s}} \cos 4\left(\varphi_{M}-\varphi_{u}\right) \\
& +\frac{2 K_{u}}{M_{s}} \cos 2\left(\varphi_{M}-\varphi_{u}\right),
\end{aligned}
$$

$$
\begin{aligned}
H_{b}= & H \cos \left(\varphi_{H}-\varphi_{M}\right)+4 \pi M_{\mathrm{eff}}+\frac{K_{4}}{2 M_{s}}\left[3+\cos 4\left(\varphi_{M}-\varphi_{u}\right)\right] \\
& +\frac{K_{u}}{M_{s}}\left[1+\cos 2\left(\varphi_{M}-\varphi_{u}\right)\right],
\end{aligned}
$$

where $M_{\text {eff }}=M_{s}-K_{\perp} / 2 \pi M_{s}$ is the effective magnetization. In the following, we also note $H_{u}=2 K_{u} / M_{s}$ and $H_{4}$ $=4 K_{4} / M_{s}$ as the in-plane uniaxial and the fourfold anisotropy fields, respectively.

In addition, the FMR and MS-FMR techniques allow observation of perpendicular standing spin waves. For a thin film of thickness $d$, the frequencies of PSSW in case of an applied magnetic field parallel to the plane of the film are given approximately by ${ }^{15}$

$$
F_{n \|}=\frac{\gamma}{2 \pi}\left(H_{n a} H_{n b}\right)^{1 / 2},
$$

with

$$
\begin{aligned}
& H_{n a}=H_{a}+\frac{2 A_{\mathrm{ex}}}{M_{s}}\left(\frac{n \pi}{d}\right)^{2}, \\
& H_{n b}=H_{b}+\frac{2 A_{\mathrm{ex}}}{M_{s}}\left(\frac{n \pi}{d}\right)^{2},
\end{aligned}
$$

where $A_{\text {ex }}$ is the exchange stiffness constant and $n$ is an integer referring to the index of the PSSW mode.

As discussed in the following, this model provides a satisfactory account of most of our experimental results. However, for the thickest sample $(83 \mathrm{~nm})$, the above-mentioned orthorhombic behavior of the anisotropy contribution is not perfectly observed. For this case, in order to improve the fit with experimental data, we used a distorted form of the energy density,

$$
\begin{aligned}
E_{\text {anis }}= & K_{\perp} \sin ^{2} \theta_{M}-\frac{1}{2}\left[1+\cos 2\left(\varphi_{M}-\varphi_{u}\right)\right] K_{u} \sin ^{2} \theta_{M} \\
& -\frac{1}{8}\left[3+\cos 4\left(\varphi_{M}-\varphi_{4}\right)\right] K_{4} \sin ^{4} \theta_{M} .
\end{aligned}
$$

$\varphi_{u}$ and $\varphi_{4}$ are, respectively, the angle of in-plane uniaxial and the fourfold anisotropies axes with the $x$ axis.

The resonance frequencies $F_{n}$ for the excited modes are given by

$$
\begin{aligned}
F_{n}^{2}= & \left(\frac{\gamma}{2 \pi}\right)^{2}\left[H \cos \left(\varphi_{H}-\varphi_{M}\right)+\frac{2 K_{4}}{M_{s}} \cos 4\left(\varphi_{M}-\varphi_{4}\right)\right. \\
& \left.+\frac{2 K_{u}}{M_{s}} \cos 2\left(\varphi_{M}-\varphi_{u}\right)+\frac{2 A_{\mathrm{ex}}}{M_{s}}\left(\frac{n \pi}{d}\right)^{2}\right]\left[H \cos \left(\varphi_{H}-\varphi_{M}\right)\right. \\
& +4 \pi M_{\mathrm{eff}}+\frac{K_{4}}{2 M_{s}}\left[3+\cos 4\left(\varphi_{M}-\varphi_{4}\right)\right] \\
& \left.+\frac{K_{u}}{M_{s}}\left[1+\cos 2\left(\varphi_{M}-\varphi_{u}\right)\right]+\frac{2 A_{\mathrm{ex}}}{M_{s}}\left(\frac{n \pi}{d}\right)^{2}\right]
\end{aligned}
$$

Indeed, if $\varphi_{4}=\varphi_{u}$ expression (8) recovers the orthorhombic form. The uniform precession mode corresponds to $n=0$ in Eqs. (7) and (9). 
In FMR experiments at fixed high frequency $(22 \mathrm{GHz}$ in our case), the resonance fields are large $(>1 \mathrm{kOe})$ and the magnetic in-plane anisotropies can be neglected. Therefore, for an out-of-plane applied magnetic field the resonance frequency of the uniform mode is given by

$$
\begin{aligned}
F_{\text {out }}^{2}= & \left(\frac{\gamma}{2 \pi}\right)^{2}\left[H \cos \left(\theta_{M}-\theta_{H}\right)-4 \pi M_{\text {eff }} \cos 2 \theta_{M}\right] \\
& \times\left[H \cos \left(\theta_{M}-\theta_{H}\right)-4 \pi M_{\text {eff }} \cos ^{2} \theta_{M}\right] .
\end{aligned}
$$

In contrast to the FMR measurements, the BLS studies generally involve spin-wave modes with a nonzero wave vector $\mathbf{q}$ parallel to the film. ${ }^{16}$ The frequencies of these modes are $\mathbf{q}$ dependent and are not given by analytic expressions. However, they can be numerically calculated. ${ }^{16}$ Moreover, for the relatively small values of $\mathbf{q}$ involved in this study, an approximate analytic expression of the frequency of the lowest quasidipolar mode can be obtained. ${ }^{17}$

\section{SAMPLES AND EXPERIMENTAL SETUPS}

\section{A. Samples}

$\mathrm{Co}_{2} \mathrm{MnGe}$ films of 34,55 , and $83 \mathrm{~nm}$ in thickness were grown on $\mathrm{Al}_{2} \mathrm{O}_{3} a$-plane substrates (showing an in-plane $c$ axis) at a temperature of $400{ }^{\circ} \mathrm{C}$ by rf sputtering at an Argon pressure of $5 \times 10^{-3}$ mbar and a rate deposition of 6 $\times 10^{-2} \mathrm{~nm} / \mathrm{s}$. Before the deposition of the Heusler film, a 4-nm-thick seed layer of vanadium (V) was grown in order to induce (110) growth of the Heusler layer. ${ }^{18}$ A final 4-nmthick protective gold overlayer was deposited on top of the film. The 34 and $55 \mathrm{~nm}$ thicknesses were measured using $\mathrm{X}$-ray $(\mathrm{XR})$ reflectometry technique providing a $\pm 2 \%$ precision; for the thickest sample $(83 \mathrm{~nm})$ it was estimated from the deposition rate. XRD experiments using a double crystal diffractometer with $\mathrm{Cu} K \alpha_{1}$ radiation (1.5405 $\AA$ ) demonstrate the good quality of the $\mathrm{Co}_{2} \mathrm{MnGe}$ film and its epitaxial relationship with the substrate. The $\theta-2 \theta$ pattern [see Fig. 2(a)] indicates that the $\mathrm{Co}_{2} \mathrm{MnGe}$ (55-nm-thick) film is (110) oriented. We find a cubic lattice constant of $5.755 \AA$, in good agreement with the previously published one. ${ }^{5,19}$ The $\mathrm{X}$-ray pole figures provide a film texture [see Fig. 2(b)] with a strongly dominant orientation of the threefold [1 $\overline{1} 1]$ and [1iㅣ] axes along the $\mathbf{c}$ rhombohedral direction ( $x$ axis). This is presumably favored by the underlying vanadium seed layer. It results that the Heusler film mostly consists of two kinds of distinct domains with a [001] axis at $\pm 54.5^{\circ}$ from the $x$ direction.

\section{B. Experimental setups}

All the experiments in this study were performed at room temperature. The static measurements are carried out using a superconducting quantum interference device (SQUID) and a MOKE setup. The SQUID data were used to derive the saturation magnetization $M_{s}$. MOKE was used to obtain hysteresis loops with various geometrical arrangementsmagnetic field applied parallel to the sample edges or perpendicular to the film plane. For the dynamic measurements, $22 \mathrm{GHz}$ conventional FMR, MS-FMR, and BLS have been used. The conventional FMR setup is described in Ref. 20. Concerning the MS-FMR, the magnetic sample is mounted on a microstrip line which excites its magnetization. This microstrip line is connected to a microwave generator (218.6 GHz) and to a Schottky detector used to measure the transmitted power. For each applied external field, the sample is swept through the resonance by varying the microwave frequency. When the sample undergoes a ferromagnetic resonance, the microwave losses are increased, changing slightly the transmitted power. In addition, the external magnetic field is modulated at a frequency of $170 \mathrm{~Hz}$ with amplitude of 4 Oe. This modulation allows lock-in detection to be used in order to increase the signal-to-noise ratio. The recorded signal is proportional to the field derivative of the imaginary part of the rf susceptibility as a function of the microwave frequency. The resonance fields (conventional FMR) and frequencies (MS-FMR) are obtained from a fit assuming a Lorentzian derivative shape of the recorded data. In contrast to the conventional FMR, the MS-FMR allows dynamic measurements over a large frequency range and low external fields can be applied in order to put in evidence small magnetic anisotropies, which are not detected with high applied magnetic fields for conventional FMR.

The BLS measurements constitute a complement to the FMR and MS-FMR experiments since they give access to spin-wave modes with nonzero wave-vector values. The spin waves traveling in the film inelastically scatter the light provided by an incident monochromatic beam. The frequency shift is analyzed using a $2 \times 3$ pass Fabry-Perot interferometer, which typically gives access to the $3-300 \mathrm{GHz}$ spectral frequency range. For the used backscattering study, the investigated wave vector lies in the plane of incidence and its amplitude is equal to $4 \pi \sin \psi / \lambda$ ( $\psi$ is the angle of incidence and $\lambda$ is the wavelength of the illuminating laser $\lambda$ $=5145 \AA$ ). Therefore, its amplitude can be swept in the $[\approx 0-20] \mu \mathrm{m}^{-1}$ interval through the rotation of the sample around a planar axis. For each $\mathrm{Co}_{2} \mathrm{MnGe}$ sample, various excited spin waves were studied versus the in-plane magnetic applied field amplitude and as function of the angle of incidence of the illuminating beam (wave vector).

\section{RESULTS AND DISCUSSION}

\section{A. Static magnetic measurements}

The saturation magnetizations of the 34 and the $55 \mathrm{~nm}$ samples were measured using the SQUID: the values obtained for $4 \pi M_{s}$ are 10600 and 10900 Oe, respectively, with an error bar of $\pm 5 \%$ mostly related to the lack of precision concerning the thickness and the surface area. These values are only slightly smaller than the measured ones in the bulk alloy (12 200 Oe). ${ }^{19}$ The hysteresis loops using an in-plane applied magnetic field along the sample edges $(x$ and $y$ axes) show a rectangular shape with coercive fields of about 5 Oe, as illustrated in Fig. 3(a) for the 55-nm-thick sample. This suggests a weak planar anisotropy with characteristic anisotropy fields in the range of tens of Oe, but our data do not allow a convincing quantitative determination of in-plane anisotropy parameters. Moreover, the hysteresis loops obtained using a magnetic field parallel to the $x$ axis 

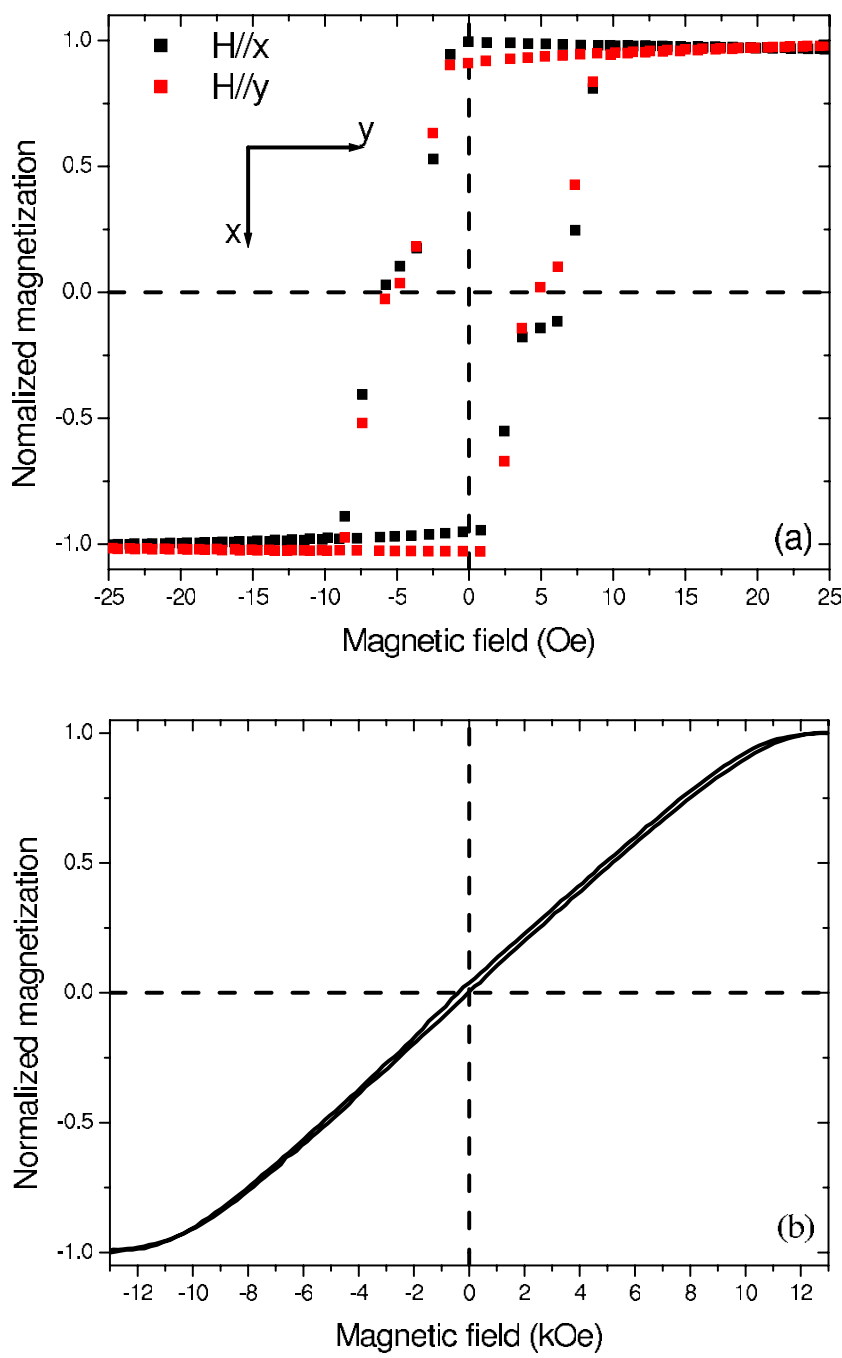

FIG. 3. (Color online) MOKE magnetization loops of the 55nm-thick $\mathrm{Co}_{2} \mathrm{MnGe}$ sample. The magnetic field is applied (a) along one of the sample edges or (b) perpendicular to the sample plane. Sketch for the direction of the applied field is indicated in figure (a).

(i.e., to the $\mathbf{c}$ axis of the corundum substrate) or to the perpendicular $y$ axis do not show significant differences: this suggests that the angle $\varphi_{u}$ introduced in Eq. (2) is equal to $45^{\circ}$. A more detailed analysis of the shape, based on the coherent rotation model, allows us to conclude that fourfold and twofold anisotropy terms present the same order of magnitude. In contrast, when the applied field is normal to the film, the magnetization loops agree with the usual scheme of a coherent rotation in the hard axis geometry, as shown in Fig. 3(b). The effective anisotropy field $H_{\text {eff }}=4 \pi M_{\text {eff }}$ is found to lay around 10000 Oe for the three studied films. More specifically, we derive 9800, 10300 , and $10000 \mathrm{Oe}$ values for the 34-, 55-, and 83-nm-thick samples, respectively. However, due to the experimentally observed rounding of the magnetization behavior in the vicinity of $H_{\mathrm{eff}}$, its determination using this technique suffers from a lack of precision. The obtained values of $4 \pi M_{s}$ and of $4 \pi M_{\text {eff }}$ are presented in Table I. Considering the poor obtainable precision the anisotropy field $H_{a \perp}$ can be only roughly estimated to lie around $1000 \mathrm{Oe}$.

\section{B. Dynamic measurements by MS-FMR, conventional FMR, and BLS}

In principle, each of the above-mentioned experimental techniques (conventional FMR, MS-FMR, and BLS) gives access to the full manifold of the magnetic coefficients, which define the energy density. However, the available precision upon the determination of a given coefficient depends on the used tool. For this reason, in a first step we have chosen to derive the value of each parameter from the bestadapted technique and, in a second step, to eventually comment on slight misfits arising from the comparison with the other techniques. Therefore, we first used the conventional FMR measurements to determine the $g$ factor and the effective magnetization since the in-plane anisotropy fields can be neglected due to the high resonance fields $(>3 \mathrm{kOe})$ at 22 GHz. Afterwards, the MS-FMR data have been used to deduce the in-plane anisotropy fields and the exchange stiffness constant. Finally, the different extracted parameters from these two techniques are used to fit the BLS data and the discrepancies between the three techniques are discussed.

Typical obtained MS-FMR and FMR spectra, where the field derivative of the microwave power absorption has been recorded, as a function of the frequency and the static magnetic field, respectively, are shown in Figs. 4(a) and 4(b) in the case of the 55-nm-thick $\mathrm{Co}_{2} \mathrm{MnGe}$ film. The MS-FMR spectrum is obtained with an in-plane field (parallel to the sample edge $x$ axis) of 280 Oe by varying the frequency in the range of $2-18 \mathrm{GHz}$ while the FMR one is obtained through the sweep of a perpendicular magnetic field in the range of 0-19.5 kOe. Both spectra display two modes where the intense peak is identified with the uniform precession mode and the second one is the first PSSW mode $[n=1$ in

TABLE I. Magnetic parameters obtained from the best fits to our experimental results. $\varphi_{u}$ and $\varphi_{4}$ are, respectively, the angles of in-plane uniaxial and of fourfold anisotropy. For the 34-nm-thick sample, the exchange constant has been determined from the BLS measurements (n.m.: not measured; extr.: extrapolated).

\begin{tabular}{|c|c|c|c|c|c|c|c|c|c|c|}
\hline $\begin{array}{c}\text { Thickness } \\
\text { (x-reflectometry) } \\
(\mathrm{nm})\end{array}$ & $\begin{array}{c}4 \pi M_{s} \\
(\mathrm{SQUID}) \\
(\mathrm{Oe})\end{array}$ & $\begin{array}{c}4 \pi M_{\text {eff }} \\
(\mathrm{MOKE}) \\
(\mathrm{Oe})\end{array}$ & $\begin{array}{c}4 \pi M_{\text {eff }} \\
(\mathrm{FMR}, \mathrm{BLS}) \\
(\mathrm{Oe})\end{array}$ & $\begin{array}{c}g \\
\text { BLS }\end{array}$ & $\begin{array}{c}g \\
\text { FMR }\end{array}$ & $\begin{array}{c}H_{u}=2 K_{u} / M_{s} \\
(\mathrm{Oe})\end{array}$ & $\begin{aligned} H_{4}= & 4 K_{4} / M_{s} \\
& (\mathrm{Oe})\end{aligned}$ & $\begin{array}{c}\varphi_{u} \\
(\mathrm{deg})\end{array}$ & $\begin{array}{c}\varphi_{4} \\
(\mathrm{deg})\end{array}$ & $\begin{array}{c}A_{\mathrm{ex}} \\
(\mathrm{erg} / \mathrm{cm})\end{array}$ \\
\hline 34 & 10600 & 9800 & 9000 & 2.1 & 2.17 & 5 & -20 & 45 & 45 & $1.07 \times 10^{-6}$ \\
\hline 55 & 10900 & 10300 & 9800 & & & 10 & -50 & 45 & 45 & $1.15 \times 10^{-6}$ \\
\hline 83 (extr.) & n.m. & 10000 & 9200 & & & 15 & 22 & -5 & 0 & $1.38 \times 10^{-6}$ \\
\hline
\end{tabular}



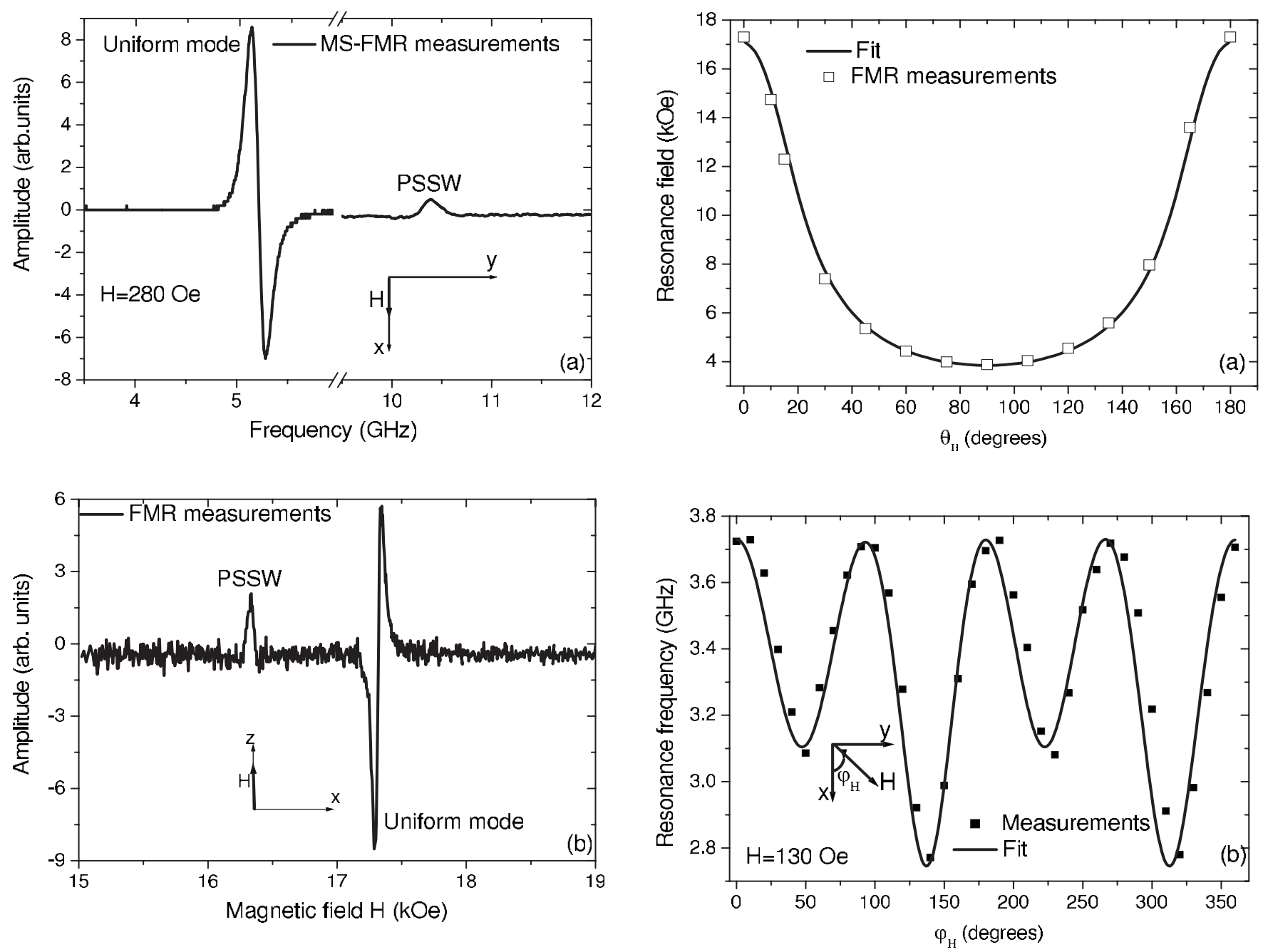

FIG. 4. (a) MS-FMR and (b) conventional FMR spectra of 55nm-thick $\mathrm{Co}_{2} \mathrm{MnGe}$ Heusler thin film for a magnetic field applied (a) parallel to the $x$ axis and (b) perpendicular to the film plane. In (a), the amplitude of the perpendicular standing spin-wave mode (PSSW) is multiplied by 10 for clarity. Sketch for the direction of the applied field is indicated in the figure.

Eq. (7)]. In our study, the MS-FMR will be used to measure the field- and the in-plane angular dependence of the resonance frequency, and the conventional FMR is devoted to the investigation of the out-of-plane angular dependence of the resonance field of the spin waves (uniform mode and PSSWs).

\section{Gyromagnetic $g$ factor and effective magnetization}

The conventional FMR technique allows deriving precise values of $g$ and $4 \pi M_{\text {eff }}$ from the variation in the resonance field of the uniform mode versus the angle $\theta_{H}$ between the applied field and the normal to the film using Eq. (10). The typical out-of-plane angular dependence of the resonance field of the uniform mode for the 55-nm-thick film measured by conventional FMR is shown in Fig. 5(a). As is typical for thin films, a dominant contribution of the shape anisotropy is observed in the out-of-plane FMR measurements. The experimental resonance field variation versus $\theta_{H}$ for this sample is in excellent agreement with the calculated ones

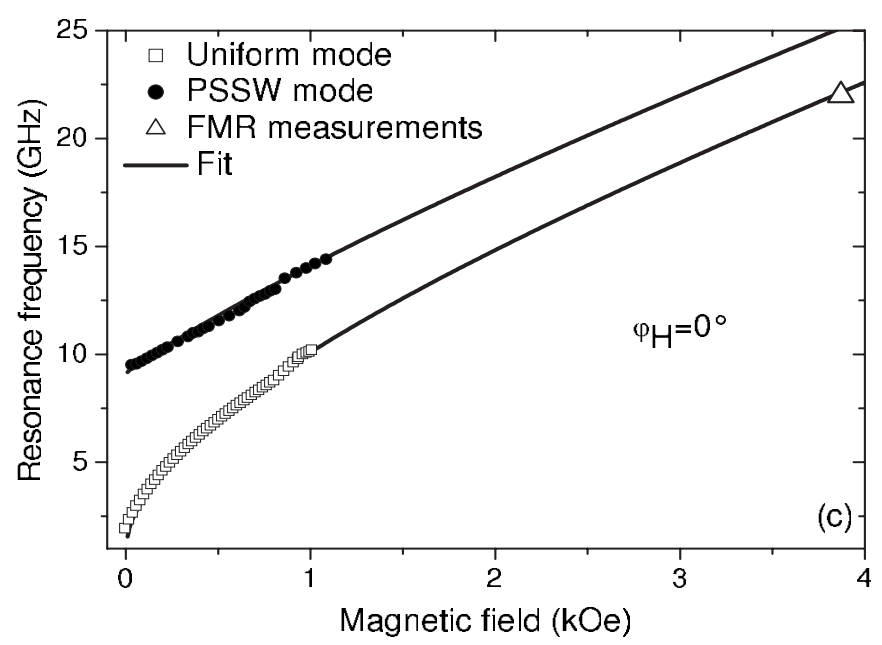

FIG. 5. (a) Conventional FMR out-of-plane angular-dependence of the resonance field, (b) MS-FMR in-plane angular dependence of the resonance frequency of the uniform mode, and (c) resonance frequency of the uniform and of the first PSSW modes as a function of the in-plane field (parallel to $x$ axis) of a 55-nm-thick $\mathrm{Co}_{2} \mathrm{MnGe}$ Heusler film. The corresponding fit is obtained using Eqs. (6), (7), and (10) with the parameters indicated in Table I. Sketch for the direction of the applied field is indicated in figure (b). 
using Eq. (10) with $g=2.17$ and $4 \pi M_{\text {eff }}=9800$ Oe. The same $g$ value is obtained with the 34- and the 83-nm-thick films, but with slightly different effective demagnetizing fields-9000 and 9200 Oe, respectively. The present $M_{\text {eff }}$ values slightly differ from the deduced ones from MOKE measurements, but this is presumably a consequence of the rather large uncertainty inherent to this last method, as pointed out in Sec. IV A. The FMR results suggest that the above evaluation of 1000 Oe for $H_{a \perp}$ is underestimated and that a value of 1500 Oe is more realistic.

\section{In-plane anisotropy}

The study of spin-wave frequencies as a function of the in-plane sample orientation allows for the determination of in-plane anisotropy constants. Therefore, the in-plane anisotropy terms were deduced from the MS-FMR study of samples subjected to a weak in-plane magnetic field (130 Oe), as a function of its direction (defined by $\varphi_{H}$ ). In Fig. 5(b), the typical experimental in-plane angular dependence of the resonance frequency of the uniform mode for the 55nm-thick sample is compared to the obtained fit using Eq. (6) with $\varphi_{u}=45^{\circ}$. The anisotropy axes are straightforward deduced from the fit of the data with Eq. (6) where the principal anisotropy directions correspond to the extrema of the resonance frequency. An excellent fit using the same $\varphi_{u}$ value is obtained with the 34-nm-thick sample but with different $H_{4}$ and $H_{u}$ parameters. These results are in agreement with the previously suggested hypothesis of principal magnetic anisotropy directions at $45^{\circ}$ of the $\mathrm{c}$ axis of the corundum substrate. However the interpretation is not straightforward since the $\mathrm{x}$-ray measurements show that, for most of the film grains, the twofold [001] crystallographic axis is inclined by $\pm 54.5^{\circ}$ from the $\mathbf{c}$ direction: assuming an equipartition of these two orientations, one would expect for an effective anisotropy energy characterized by a principal axis along c. The values of $H_{4}$ and $H_{u}$ are reported in Table I. Notice that the tetragonal term is significantly higher than the uniaxial one, which maybe reflects a partial averaging due to the occurrence of orthogonal domains. Also remark that $H_{4}$ is negative, which means that, in the absence of $H_{u}$, the easy in-plane tetragonal axis would be the $\mathbf{c}$ axis of the corundum substrate. The small values of the in-plane anisotropy terms induce small variations in the resonance frequency for high applied fields and, consequently, as mentioned above, do not significantly affect the FMR measurements realized under high applied magnetic fields.

The 83-nm-thick sample presents a different in-plane angular dependence of the resonance frequency compared to the 34- and 55-nm-thick samples (Fig. 6). Only an approximate fit can be obtained [Fig. 6(a)]: it corresponds to $\varphi_{u}$ $=0^{\circ}$, to compare with $\varphi_{u}=45^{\circ}$ in the preceding cases. One of the principal anisotropy axes looks now parallel to the $\mathbf{c}$ axis of the substrate, in agreement with the behavior expected from the crystallographic texture. Notice that $H_{4}$ is now positive, which means that in the absence of $H_{u}$, the easy inplane tetragonal principal direction would be the same as for the 34- and 55-nm-thick samples, i.e., the c axis of the corundum substrate. The uniaxial in-plane character is more marked than for the thinner samples, as attested by the larger
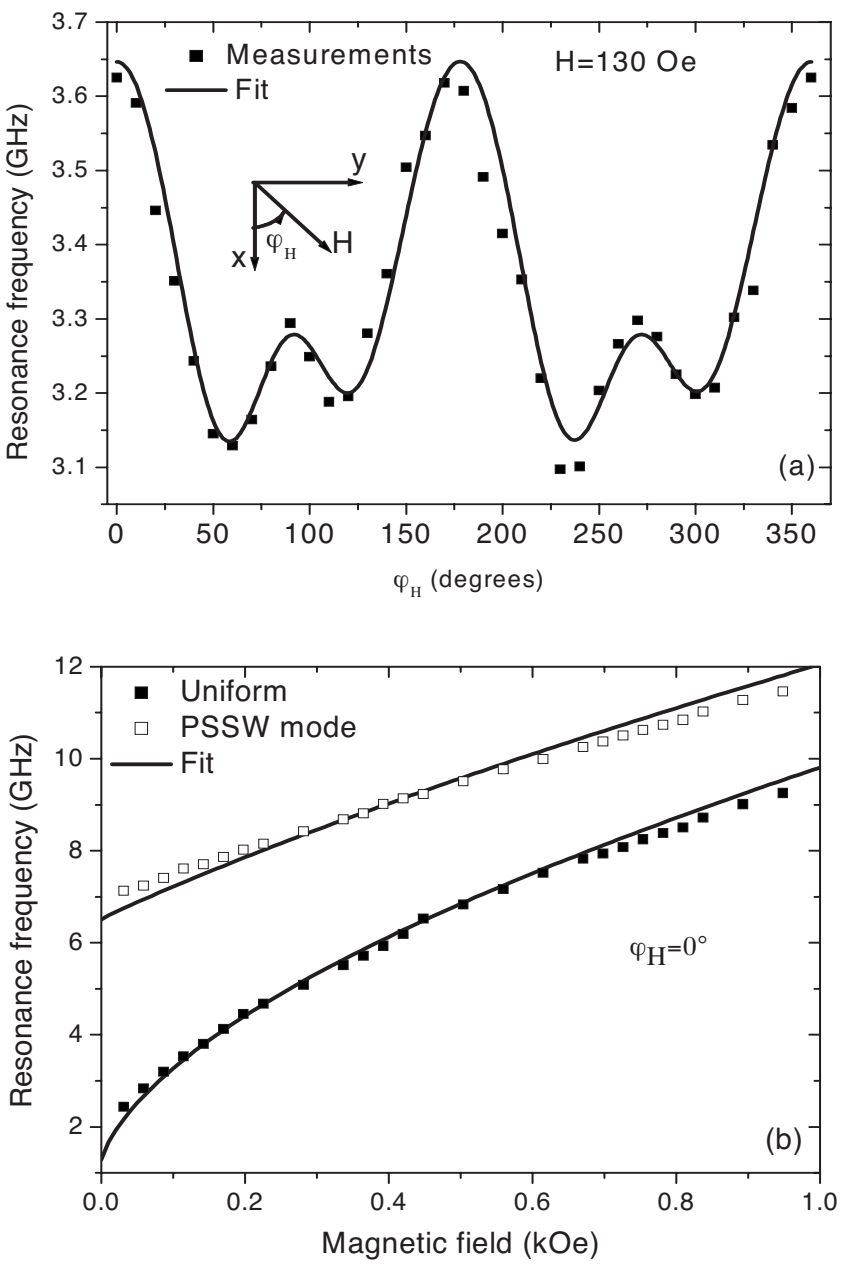

FIG. 6. (a) MS-FMR in-plane angular dependence of the resonance frequency of the uniform mode and (b) resonance frequency of the uniform and the first PSSW modes as a function of the in-plane field (parallel to sample edge: $x$ axis) of 83-nm-thick $\mathrm{Co}_{2} \mathrm{MnGe}$ film. The corresponding fit is obtained using Eqs. (6) and (7) with the parameters indicated in Table I. Sketch for the direction of the applied field is indicated in figure (a).

value of $H_{u} / H_{4}$. Finally, as seen in Fig. 6(a), a completely satisfying fit can be found by choosing $\varphi_{4}=0^{\circ}$ and $\varphi_{u}=-5^{\circ}$ : the $5^{\circ}$ difference between $\varphi_{4}$ and $\varphi_{u}$ gives account of the loss of symmetry of the frequency variation around $\varphi_{H}=90^{\circ}$. This behavior of the magnetic anisotropy direction is most probably induced by a modification of the growth direction as the thickness becomes higher and more XR investigations would be needed to interpret this behavior.

\section{Exchange stiffness constant and BLS measurements}

All the magnetic parameters extracted from the above studies are now used to fit the field-dependence frequency of the uniform precession mode and of the first PSSW mode (when it can be detected by our MS-FMR setup-55- and 83-nm-thick films) using Eqs. (7) and (9) for an applied magnetic field parallel to the $x$ axis [Figs. 5(c) and 6(b)]. This 


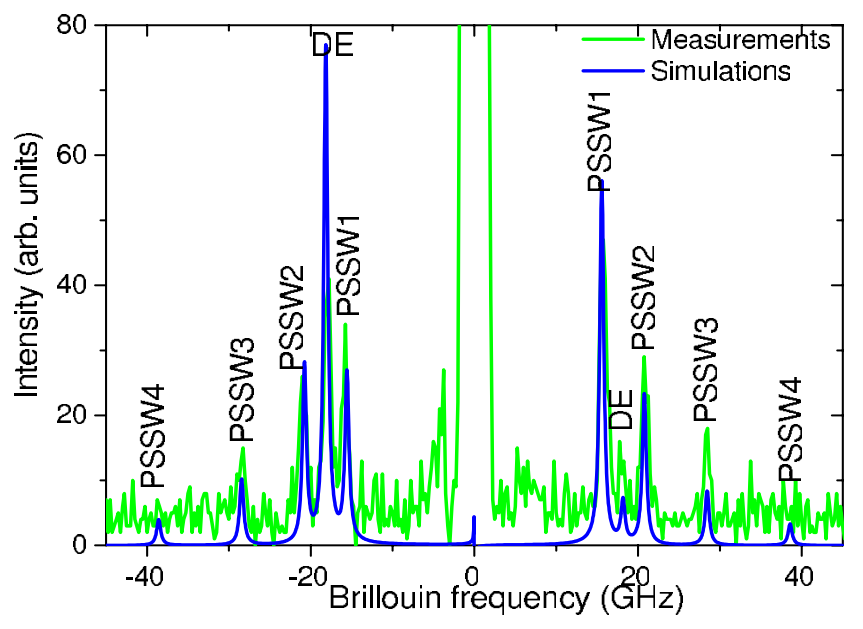

FIG. 7. (Color online) Example of BLS spectrum measured on the 83-nm-thick $\mathrm{Co}_{2} \mathrm{MnGe}$ Heulser film in an external magnetic field of $2 \mathrm{kOe}$. The magnetic field is applied parallel to the film (parallel to $x$ axis) and perpendicular to the plane of light incidence. Negative frequencies are related to Stokes processes (creation of magnons), whereas positive frequencies are related to anti-Stokes processes (annihilation of magnons). The corresponding simulations are obtained using our model presented in Ref. 16 using $A_{\mathrm{ex}} / M_{s}$ $=1.27 \times 10^{-9} \mathrm{erg} \mathrm{cm}^{-1} \mathrm{Oe}^{-1}, \quad g=2.1, \quad$ a wave vector $q=6.3$ $\times 10^{4} \mathrm{~cm}^{-1}$, a sample thickness of $83 \mathrm{~nm}, 4 \pi M_{\text {eff }}=9200 \mathrm{Oe}$, $4 \pi M_{s}=10900$ Oe, and other parameters indicated in Table I.

allows to check the validity of the above extracted magnetic parameters and provides an evaluation of the exchange stiffness constant $A_{\text {ex }}$, which is an important parameter from both fundamental and application points of view. More precisely, this experimental protocol allows us to evaluate $A_{\mathrm{ex}} /\left(M_{s} d^{2}\right)$. Unfortunately, due to the weakness of the signal related to the PSSW mode, this technique did not allow extracting the exchange term using the thinnest $(34 \mathrm{~nm})$ film. The best fit to the experimental data of the 55-nm film [Figs. 5(c) and 6(b)] was obtained for $A_{\mathrm{ex}} /\left(M_{s} d^{2}\right)=43.7 \mathrm{erg} \mathrm{cm}^{-3} \mathrm{Oe}^{-1}$, which leads to a $1.15 \times 10^{-6} \mathrm{erg} \mathrm{cm}^{-1}$ value of $A_{\text {ex }}$. Assuming the same saturation magnetization for the $83-\mathrm{nm}$ film we find $A_{\mathrm{ex}}=1.38 \times 10^{-6} \mathrm{erg} \mathrm{cm}^{-1}$ for the $83-\mathrm{nm}$ film. These values also fit with the results obtained for conventional FMR with an applied field perpendicular to the film. The difference between the two calculated values arises from the limited precision upon the thickness and the saturation magnetization especially for the 83-nm-thick sample.

Let us now analyze the BLS spectra, which were recorded using an in-plane relatively large applied field (above 500 Oe). In such cases, the in-plane anisotropy terms can be neglected and the spectra practically only depend upon $g, M_{\text {eff }}$, $q d$, and $\left(A_{\mathrm{ex}} / M_{s} d^{2}\right)$. In order to record spectra related to a large number of PSSWs, we mainly focused our study on the 83-nm-thick sample. A typical spectrum concerning this sample, with a 2 kOe in-plane applied field parallel to the $x$ axis and perpendicular to the wave vector $\mathbf{q}(q=6.3$ $\left.\times 10^{4} \mathrm{~cm}^{-1}\right)$ is shown in Fig. 7. It contains a rich structure including the pseudodipolar Damon-Eschbach mode (originating from the uniform mode which corresponds to the zero-wave-vector case, i.e., to normal incidence in BLS backscattering) and several PSSW. Notice that the maxima

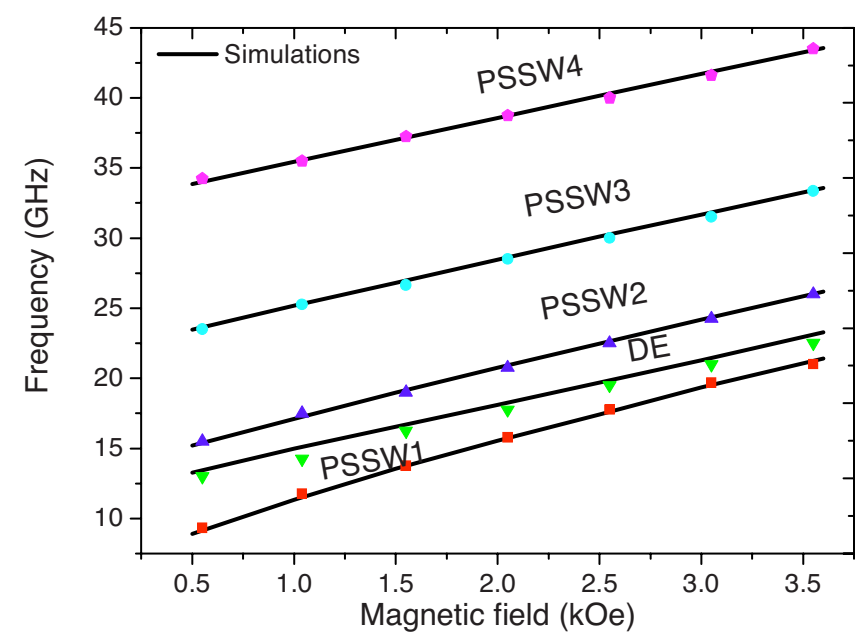

FIG. 8. (Color online) Dependence of BLS frequencies on the external magnetic field for $83-\mathrm{nm}$-thick $\mathrm{Co}_{2} \mathrm{MnGe}$ film. The magnetic field is applied parallel to the film (parallel to $x$ axis) and perpendicular to the plane of light incidence. Symbols indicate experimental data and lines refer to the simulation according to our model presented on Ref. 16 using $A_{\mathrm{ex}} / M_{s}=1.27$ $\times 10^{-9} \mathrm{erg} \mathrm{cm}^{-1} \mathrm{Oe}^{-1}, g=2.1$, a wave vector $q=6.3 \times 10^{4} \mathrm{~cm}^{-1}$, a sample thickness of $83 \mathrm{~nm}, 4 \pi M_{\text {eff }}=9200$ Oe, $4 \pi M_{s}=10900$ Oe, and other parameters indicated in Table I.

directly provide the frequencies of the involved spin waves in the scattering process. The shape of the spectrum can be numerically calculated, ${ }^{16}$ thus allowing to fit the spectra with appropriate parameters. Figure 8 shows the experimental frequency variations versus the applied field compared to our best fit, obtained using the above cited values of the thickness and of the magnetic parameters $M_{\mathrm{eff}}, M_{s}$, and $A_{\mathrm{ex}}$ but a slightly smaller $g$ coefficient $(g=2.1$ instead of 2.17). For the two above-discussed samples $(55$ and $83 \mathrm{~nm}$ ) the agreement between calculated and observed spectra is satisfying using this reduced value of $g$ and the previously obtained values of $4 \pi M_{\text {eff }}, M_{s}$, and $A_{\text {ex }}$ (see Table I). In addition, the BLS data allowed us to calculate the exchange stiffness constant related to 34-nm-thick sample- $A_{\mathrm{ex}}=1.07 \times 10^{-6} \mathrm{erg} / \mathrm{cm}$. The weak discrepancy for $g$ seems to overpass the experimental uncertainty and remains to be interpreted.

\section{SUMMARY}

MS-FMR, conventional FMR, and BLS have been used to study the magnetic dynamic properties of sputtered 34-, 55-, and 83-nm-thick $\mathrm{Co}_{2} \mathrm{MnGe}$ films. The behavior of the 34and 55-nm-thick films is described assuming a magnetic energy density monitored by an orthorhombic symmetry with one twofold axis normal to the film and the two planar remaining ones at $\pm \pi / 4$ of the $\mathbf{c}$ axis of the substrate; however, the comparative deduced values of the pertinent coefficients show that this behavior does not much deviate from the derived one assuming a tetragonal symmetry. As the $\mathrm{Co}_{2} \mathrm{MnGe}$ films become thicker $(83 \mathrm{~nm})$, the in-plane anisotropies axes rotate by $45^{\circ}$; however, such a situation, 
which is the expected one, is slightly perturbed by a small misalignment of $5^{\circ}$ between the uniaxial and the fourfold anisotropy axes. Good agreement between BLS, MS-FMR measurements, and the simulations is found. The fast magnetization dynamics in such compounds and other Heuslers will be addressed in future work.

\section{ACKNOWLEDGMENTS}

The authors would like to thank C. Chappert and T. Devolder for putting at their disposal the experimental MOKE setup. T. Chauveau and D. Faurie are also acknowledged for the $\mathrm{X}$-ray measurements during this study.
${ }^{1}$ S. Picozzi, A. Continenza, and A. J. Freeman, Phys. Rev. B 66, 094421 (2002).

${ }^{2}$ S. Picozzi, A. Continenza, and A. J. Freeman, J. Phys. Chem. Solids 64, 1697 (2003).

${ }^{3}$ T. Ambrose, J. J. Krebs, and G. A. Prinz, J. Appl. Phys. 89, 7522 (2001).

${ }^{4}$ T. Ishikawa, T. Marukame, K. Matsuda, T. Uemura, M. Arita, and M. Yamamoto, J. Appl. Phys. 99, 08J110 (2006).

${ }^{5}$ F. Y. Yang, C. H. Shang, C. L. Chien, T. Ambrose, J. J. Krebs, G. A. Prinz, V. I. Nikitenko, V. S. Gornakov, A. J. Shapiro, and R. D. Shull, Phys. Rev. B 65, 174410 (2002).

${ }^{6}$ H. Wang, A. Sato, K. Saito, S. Mitani, K. Takanashi, and K. Yakushiji, Appl. Phys. Lett. 90, 142510 (2007).

${ }^{7}$ Y. Sakuraba, M. Hattori, M. Oogane, Y. Ando, H. Kato, A. Sakuma, T. Miyazaki, and H. Kubota, Appl. Phys. Lett. 88, 192508 (2006).

${ }^{8}$ T. Marukame, T. Ishikawa, K. Matsuda, T. Uemura, and M. Yamamoto, Appl. Phys. Lett. 88, 262503 (2006).

${ }^{9}$ D. Ebke, J. Schmalhorst, N.-N. Liu, A. Thomas, G. Reiss, and A. Hütten, Appl. Phys. Lett. 89, 162506 (2006).
${ }^{10}$ J. Hamrle, S. Blomeier, O. Gaier, B. Hillebrands, R. Schäfer, and M. Jourdan, J. Appl. Phys. 100, 103904 (2006).

${ }^{11}$ J. C. Slonczewski, J. Magn. Magn. Mater. 159, L1 (1996).

${ }^{12}$ M. Farle, Rep. Prog. Phys. 61, 755 (1998).

${ }^{13}$ O. Acher, S. Queste, M. Ledieu, K.-U. Barholz, and R. Mattheis, Phys. Rev. B 68, 184414 (2003).

${ }^{14}$ K. Lenz, E. Kosubek, K. Baberschke, H. Wende, J. Herfort, H.-P. Schönherr, and K. H. Ploog, Phys. Rev. B 72, 144411 (2005).

${ }^{15}$ A. Martins, S. C. Trippe, A. D. Santos, and F. Pelegrini, J. Magn. Magn. Mater. 308, 120 (2007).

${ }^{16}$ Y. Roussigné, F. Ganot, C. Dugautier, P. Moch, and D. Renard, Phys. Rev. B 52, 350 (1995).

${ }^{17}$ F. Zighem, Y. Roussigné, S. M. Chérif, and P. Moch, J. Phys.: Condens. Matter 19, 176220 (2007).

${ }^{18}$ U. Geiersbach, K. Westerholt, and H. Bach, J. Magn. Magn. Mater. 240, 546 (2002).

${ }^{19}$ P. G. Webster, J. Phys. Chem. Solids 32, 1221 (1971).

${ }^{20}$ I. Neudecker, G. Woltersdorf, B. Heinrich, T. Okuno, G. Gubbiotti, and C. H. Back, J. Magn. Magn. Mater. 307, 148 (2006). 